\title{
Is Epileptogenic Cortex Truly Hypometabolic on Interictal Positron Emission Tomography?
}

\author{
Csaba Juhász, MD, ${ }^{*} \dagger$ Diane C. Chugani, PhD, ${ }^{*} \ddagger$ Otto Muzik, PhD, $\ddagger$ Craig Watson, MD, PhD, $\dagger$ \\ Jagdish Shah, MD, $\dagger$ Aashit Shah, MD, $\dagger$ and Harry T. Chugani, MD*†‡
}

Positron emission tomography (PET) of glucose metabolism is often applied for the localization of epileptogenic brain regions, but hypometabolic areas are often larger than or can miss epileptogenic cortex in nonlesional neocortical epilepsy. The present study is a three-dimensional brain surface analysis designed to demonstrate the functional relation between glucose PET abnormalities and epileptogenic cortical regions. Twelve young patients (mean age, 10.8 years) with intractable epilepsy of neocortical origin underwent chronic intracranial electroencephalographic monitoring. The exact location of the subdural electrodes was determined on high-resolution three-dimensional reconstructed magnetic resonance imaging scan volumes. The electrodes were classified according to their locations over cortical areas, which were defined as hypometabolic, normometabolic, or at the border between hypometabolic and normal cortex (metabolic "border zones") based on interictal glucose PET. Electrodes with seizure onset were located over metabolic border zones significantly more frequently than over hypometabolic or normometabolic regions. Seizure spread electrodes also more frequently overlay metabolic border zones than hypometabolic regions. These findings suggest that cortical areas with hypometabolism should be interpreted as regions mostly not involved in seizure activity, although epileptic activity commonly occurs in the surrounding cortex. This feature of hypometabolic cortex is remarkably similar to that of structural brain lesions surrounded by epileptogenic cortex. Cortical areas bordering hypometabolic regions can be highly epileptogenic and should be carefully assessed in presurgical evaluations.

Juhász C, Chugani DC, Muzik O, Watson C, Shah J, Shah A, Chugani HT. Is epileptogenic cortex truly hypometabolic on interictal positron emission tomography? Ann Neurol 2000;48:88-96

In patients with intractable epilepsy without structural brain lesions, the success of epilepsy surgery highly depends on the accurate presurgical delineation of the cortex responsible for generating epileptic seizures. 2-Deoxy-2- $\left[{ }^{18} \mathrm{~F}\right]$ fluoro-D-glucose (FDG) positron emission tomography (PET) is widely used to define epileptogenic cortical areas so as to replace or guide intracranial electrode placement. It is now generally accepted that focal areas of interictal glucose hypometabolism correspond to seizure foci defined by means of electroencephalography (EEG). ${ }^{1-5}$ Hypometabolic areas are commonly larger than corresponding ictal EEG abnormalities, ${ }^{6,7}$ and FDG-PET fails to detect metabolic abnormalities consistent with epileptogenic regions in 20 to $30 \%$ of patients with seizures of extratemporal origin. ${ }^{7-9}$

In a recent study of patients with extratemporal lobe epilepsy, we were able to directly compare the threedimensional (3D) brain surface location of ictal seizure onset and early spread as defined by invasive EEG monitoring with cortical areas of abnormal glucose metabolism and in vivo $\left[{ }^{11} \mathrm{C}\right]$ flumazenil binding. ${ }^{9}$ Surprisingly, we found that FDG-PET not only missed the seizure onset zone in some cases but also was not sensitive in detecting areas of rapid seizure spread. Indeed, the clinical usefulness of FDG-PET for locating the precise epileptic focus in extratemporal lobe epilepsy seemed to be modest. During the analysis of our data, however, we frequently observed that electrodes with ictal involvement were located close to areas objectively determined to be hypometabolic.

Based on this observation, we pursued an analysis in which metabolic "border zones," that is, cortical areas at the border of a hypometabolic and normometabolic region, were specifically addressed in terms of their electrophysiological activity during seizures. We hypothesized that border zones rather than sites within a hypometabolic region may harbor the epileptic focus
From the Departments of *Pediatrics, $\uparrow$ Neurology, and $\ddagger$ Radiology, Children's Hospital of Michigan Detroit Medical Center, Wayne State University School of Medicine, Detroit, MI.

Received Dec 22, 1999, and in revised form Mar 15, 2000. Accepted for publication Mar 15, 2000.
Address correspondence to Dr Chugani, Pediatric Neurology/PET Center, Children's Hospital of Michigan, 3901 Beaubien Boulevard, Detroit, MI 48201. 
and areas receiving ictal propagation in neocortical epilepsy. This concept, if true, might explain previous conflicting results and also could influence clinical interpretation of FDG-PET abnormalities.

\section{Subjects and Methods}

\section{Subjects}

Twelve young patients ( 7 boys and 5 girls; mean age, 10.8 years; Table 1) were included in the study according to the following inclusion criteria: (1) medically intractable epilepsy of neocortical origin based on seizure semiology and ictal EEG studies and (2) focal cortical areas of decreased glucose metabolism on the interictal FDG-PET scan not associated with focal cortical structural abnormalities on corresponding high-resolution magnetic resonance imaging (MRI) scans. All patients with temporal EEG foci underwent MRI volumetry $^{10}$ showing normal hippocampal and amygdaloid volumes. All patients subsequently underwent resective surgery of the seizure focus, and histological assessment was used to confirm the lack of a foreign tissue lesion or other major structural abnormalities. Postoperative outcome (classes I-IV) was determined according to the criteria of Engel and co-workers. ${ }^{11}$ All studies were performed in accordance with the regulations of the Human Investigation Committee at Wayne State University.

\section{Chronic Subdural EEG Monitoring and Analysis of EEG Data}

Subdural electrode placement was guided by the seizure semiology, with the seizure onset area determined by scalp ictal EEG and the location of FDG-PET abnormalities. In the 12 patients, a total of 788 subdural electrodes were placed covering the presumed epileptic cortex. The analysis of intracranial EEG data was performed as described previously. ${ }^{9}$ In short, at least three (but typically $>10$ ) habitual seizures were captured in every case and analyzed during in- tracranial video-EEG monitoring. The electrodes were identified as follows: electrodes indicating seizure onset (defined as a localized, sustained, rhythmic or semirhythmic, or spiking EEG pattern with a frequency of $>2 \mathrm{~Hz}$, which was visually distinguished from background activity and not attributed to the state of arousal), electrodes involved in early seizure spread (defined as an electrode involved in seizure activity within 10 seconds of seizure onset), and electrodes without early ictal involvement.

\section{PET, MRI, and Radiographic Procedures}

These were performed as described previously. ${ }^{9}$ PET studies were performed as part of the clinical presurgical evaluation using the EXACT/HR positron emission tomograph (CTI/ Siemens, Knoxville, TN) located at Children's Hospital of Michigan. This scanner generates 47 image planes with a slice thickness of $3.125 \mathrm{~mm}$. The reconstructed image inplane resolution obtained was $5.5 \pm 0.35 \mathrm{~mm}$ at full-width at half maximum. All patients had interictal PET studies as verified by EEG monitoring during the tracer uptake period.

All patients underwent a volumetric spoiled gradient (SPGR) echo MRI scan (with 124 contiguous 1.5 -mm thick sections) before subdural grid placement. Three patients ( $\mathrm{Pa}-$ tients 1, 4, and 12) also underwent MRI with the intracranial electrodes in place. Because the metal electrodes cause local artifacts in the images, the position of individual electrodes relative to the brain surface could be reliably determined in three orthogonal views of the MRI SPGR echo sequence. The position of each subdural electrode in the 3D view was then computed and displayed on top of the $3 \mathrm{D}$ brain surface obtained from the original volumetric SPGR echo sequence. In the remaining 9 patients, electrode positions were determined using a planar radiograph as described previously. ${ }^{9,12}$ Three metallic fiducial markers were placed at standard locations on the patient's head, and a planar radiographic image was acquired with the subdural electrodes in

Table 1. Clinical Data of the Patients

\begin{tabular}{|c|c|c|c|c|c|c|}
\hline $\begin{array}{l}\text { Patient No./ } \\
\text { Sex/Age (yr) }\end{array}$ & Seizure Type & $\begin{array}{l}\text { Seizure } \\
\text { Onset }\end{array}$ & $\begin{array}{l}\text { Seizure } \\
\text { Spread }\end{array}$ & $\begin{array}{l}\text { FDG-PET } \\
\text { Hypometabolism }\end{array}$ & $\begin{array}{l}\text { Interictal EEG during PET } \\
\text { and Location of } \\
\text { Epileptiform Activity }\end{array}$ & $\begin{array}{l}\text { Postsurgical } \\
\text { Follow-Up/ } \\
\text { Outcome }\end{array}$ \\
\hline $1 / F / 13$ & CP, GTC & LF & $\mathrm{LF}, \mathrm{T}$ & $\mathrm{LF}, \mathrm{T}, \mathrm{P}$ & $\mathrm{BiF}(\mathrm{L}>\mathrm{R}), \mathrm{L} T$ & $17 \mathrm{mo} / \mathrm{I}$ \\
\hline $2 / F / 15$ & SP, CP & R F & R F, T & R F, T, P & R F-T & $13 \mathrm{mo} / \mathrm{IV}^{\mathrm{a}}$ \\
\hline $3 / \mathrm{M} / 6$ & sGTC & R F & R F, T, P & R C, T, P & $\mathrm{R} \mathrm{T}$ & $24 \mathrm{mo} / \mathrm{II}$ \\
\hline $4 / F / 2$ & SP & $\mathrm{LC}$ & LF & LF, T, P & No epileptiform activity & $20 \mathrm{mo} / \mathrm{III}^{\mathrm{a}}$ \\
\hline $5 / \mathrm{M} / 10$ & CP, GTC & L C & LF & LF, T, P & BiT-P & $15 \mathrm{mo} / \mathrm{I}$ \\
\hline $6 / \mathrm{M} / 16$ & $\mathrm{CP}$ & R F & R F, T & R F, T, P & R F-T & $17 \mathrm{mo} / \mathrm{I}$ \\
\hline 7/M/19 & SP, CP, GTC & $\mathrm{LT}$ & LF & $\mathrm{LF}, \mathrm{T}$ & Generalized, $\mathrm{L}>\mathrm{R}$ & $16 \mathrm{mo} / \mathrm{III}^{\mathrm{a}}$ \\
\hline $8 / \mathrm{M} / 18$ & SP, CP, GTC & $\mathrm{LT}$ & $\mathrm{L} T, \mathrm{~F}$ & $\mathrm{~L} T, \mathrm{~F}$ & $\mathrm{LT}$ & $11 \mathrm{mo} / \mathrm{I}$ \\
\hline $9 / F / 3.5$ & $\mathrm{CP}$ & $\mathrm{LT}$ & LF & $\mathrm{LT}, \mathrm{P}, \mathrm{F}$ & L T-F & $8 \mathrm{mo} / \mathrm{I}$ \\
\hline $10 / F / 4.5$ & SP, CP & $\mathrm{LC}$ & $\mathrm{L} \mathrm{T}$ & $\mathrm{LC}$ & L C & $7 \mathrm{mo} / \mathrm{I}$ \\
\hline $11 / \mathrm{M} / 4$ & $\begin{array}{l}\mathrm{CP} \text {, atonic } \\
\text { myoclonic }\end{array}$ & L T-P & - & L T-P, F & L F-T; R P-T, O & $13 \mathrm{mo} / \mathrm{I}$ \\
\hline $12 / \mathrm{M} / 19$ & SP, GTC & LF & $\mathrm{L} T, \mathrm{P}$ & LF, T & No epileptiform activity & $23 \mathrm{mo} / \mathrm{I}$ \\
\hline
\end{tabular}

${ }^{a}$ Epileptogenic cortex could be incompletely resected because it partially involved eloquent cortex (motor or speech areas).

FDG = 2-deoxy-2- $\left[{ }^{18} \mathrm{~F}\right]$ fluoro-D-glucose; $\mathrm{PET}=$ positron emission tomography; EEG = electroencephalography; $\mathrm{F}=\mathrm{female} ; \mathrm{M}=$ male; $\mathrm{L}=$ left; $\mathrm{R}=$ right; $\mathrm{SP}=$ simple partial seizures; $\mathrm{CP}=$ complex partial seizures; $\mathrm{sGTC}=$ secondarily generalized tonicoclonic seizures; $\mathrm{F}=$ frontal; $\mathrm{T}=$ temporal; $\mathrm{P}=$ parietal; $\mathrm{O}=$ occipital; $\mathrm{C}=$ central. 
place. The radiograph was then digitized, and the three fiducial markers were identified on it as well as on the corresponding 3D reconstructed MRI volume, using the 3D-Tool software package. ${ }^{13}$ An iterative algorithm minimized the differences between the two sets of coordinate triplets by adjusting the three Euler angles and the image zoom., ${ }^{9}$ As a result, a cortical surface view was created, allowing the location of electrodes to be directly defined on the MRI 3D brain surface. The accuracy of the coregistration procedure between the subdural EEG electrodes and the MRI volume was reported to be $1.24 \pm 0.66 \mathrm{~mm}$, with a maximal misregistration of $2.7 \mathrm{~mm}^{12}$

\section{PET/MRI Coregistration}

PET and MRI SPGR echo volumes were coregistered as described in detail previously, ${ }^{14}$ using MPI-Tool, ${ }^{15}$ a multipurpose $3 \mathrm{D}$ registration technique developed at the Max-PlanckInstitute in Cologne, Germany. The coregistration method is highly interactive and is based on the simultaneous alignment of PET/MRI contours, which are exchanged in three orthogonal cuts through the brain. This procedure does not require external landmarks and can be used despite alterations in normal brain anatomy. Validation studies ${ }^{15}$ showed that misalignment was always smaller than the PET image resolution, and the average displacement between PET and MRI scans was reported to be less than $0.5 \mathrm{~mm} .{ }^{15}$

\section{Objective PET Image Analysis}

The extent of regional cortical abnormalities of brain glucose metabolism were identified using an objective method applied to all supratentorial planes of the PET studies. ${ }^{16}$ This procedure allows the definition of abnormal cortical areas of glucose metabolism based on asymmetry measures derived from homotopic cortical areas according to a predefined cutoff threshold. The smallest possible abnormality defined in the PET images using our objective method to detect cortical asymmetries consisted of at least three adjacent segments in two consecutive planes yielding an area of about $1 \mathrm{~cm}^{2}$.

Cortical areas with greater than $10 \%$ relative hypometabolism were "marked" by assigning the two times of the maximum value in the data set. The cutoff threshold is consistent with a normal mean asymmetry plus $1 \mathrm{SD}$ as obtained in normal groups of both children and adults. ${ }^{16}$ The marked PET file was projected onto the brain surface automatically segmented from MRI data by reverse-gradient fusion ${ }^{17}$ using the $3 \mathrm{D}$-Tool software package. ${ }^{13}$ This procedure resulted in visualization of the cortical surface with marked colorcoded FDG-PET abnormalities and superimposed subdural electrodes.

\section{Determination of the Spatial Relation between Subdural Electrodes and Glucose PET Abnormalities}

All electrodes were classified as normometabolic, hypometabolic, or border zone electrodes. Electrodes have a diameter of $5 \mathrm{~mm}$ and overlie 37 pixels on the reconstructed brain surface. Electrodes were classified as being hypo- or normometabolic when at least $90 \%$ of their pixels, or 34 pixels per electrode, were overlapping with a hypometabolic or normometabolic area, respectively. Otherwise, they were classified as being border zone electrodes (Fig 1). Furthermore, as a special subgroup of normometabolic electrodes, we determined the location of all ictally involved (onset and spread) normometabolic electrodes situated adjacent to border zone electrodes. These electrodes as well as all ictally involved electrodes adjacent to such electrodes were called "adjacent ictal" electrodes. Together with border zone electrodes, they represented a group of electrodes bordering on but not included in a hypometabolic area (see Fig 1).

\section{Data Analysis}

In each patient, the number of electrodes classified as hypometabolic, normometabolic, or border zone was calculated. The electrodes with seizure onset and seizure spread were then counted in each metabolic group separately, and this number was expressed as a percentage of the total number of electrodes in that metabolic group. This provided a percentage of occurrence of electrodes involved in seizure onset or spread for all three metabolic zones in each patient. Analysis of variance was used to determine if the mean percentage of ictal involvement (seizure onset, early spread) among electrodes located over hypometabolic, normometabolic, or border zone cortical regions was significantly different. An unpaired $t$ test was used to determine if the occurrence of the

Fig 1. Schematic for classification of subdural grid electrodes according to their spatial relation to the metabolic status of the underlying cortex: electrodes located over an interictally hypometabolic cortex (hypometabolic electrodes) (black circles); electrodes overlapping the border of a hypometabolic and normometabolic cortex (metabolic border zone electrodes) (crossed circles); and electrodes overlying normometabolic cortex (normometabolic electrodes without ictal involvement or with ictal involvement [adjacent ictal electrodes]) (white circles). The figure demonstrates that ictally involved (here, ictal onset) electrodes overlying the metabolic border zone together with adjacent ictal electrodes represent a group of electrodes adjacent to but not included in the hypometabolic region.

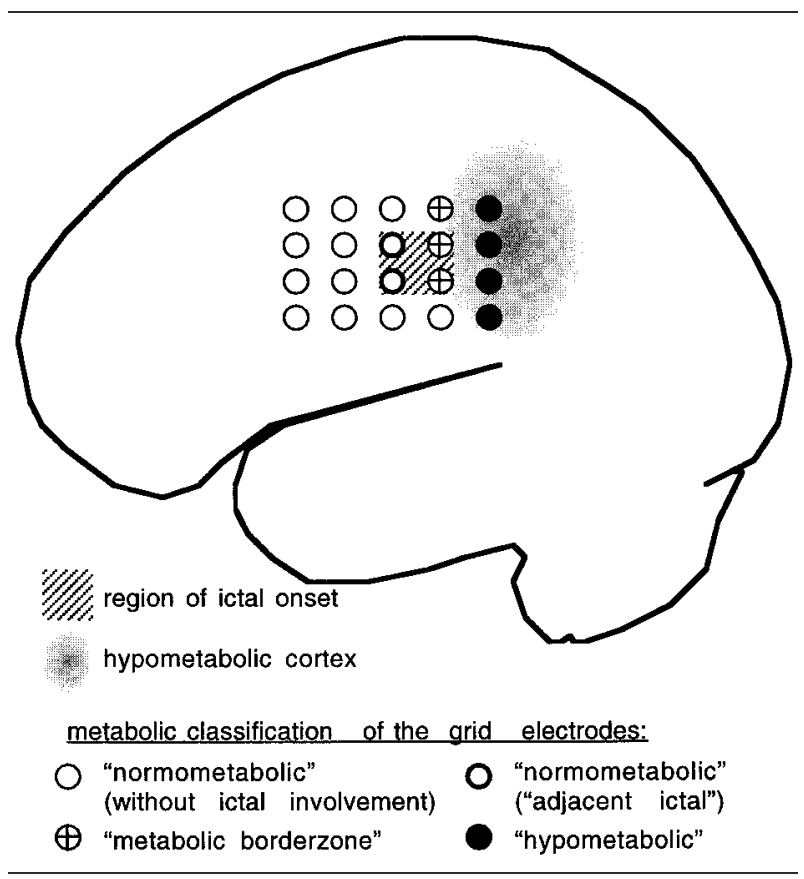


onset and spread electrodes in a particular metabolic group significantly differed. A probability value less than 0.05 was considered to be significant.

\section{Results}

In the 12 patients, a total of 26 hypometabolic regions were covered fully or partially by subdural electrodes. All three types of metabolic classification of electrodes occurred in 10 patients. None of the electrodes were classified as hypometabolic in 2 cases, but several electrodes overlay metabolic border zones in these patients.

Of a total of 788 electrodes analyzed, 235 (29.8\%) were ictally involved (including 90 with seizure onset and 145 with early spread; Table 2). Such electrodes occurred most frequently over metabolic border zones (105 of 235 electrodes [44.7\%]) or were identified as adjacent ictal electrodes over normometabolic regions (65 of 235 electrodes [27.6\%]) (illustrated in 2 cases; see Figs 2 and 3). Electrodes that did not show ictal activity occurred mostly over normometabolic areas (362 of 553 electrodes [65.5\%]).

A summary of the occurrence of electrodes with seizure onset and spread according to metabolic zones is shown for each patient in Table 2. Ictal onset occurred in electrodes overlying metabolic border zones significantly more frequently than in electrodes overlying hypo- or normometabolic regions (26 vs $8.9 \%, p=$ $0.003 ; 26$ vs $4.6 \%, p=0.0002$, respectively). Early seizure spread also occurred in electrodes overlying metabolic border zones more often than in electrodes overlying hypometabolic regions $(26.2$ vs $7.1 \%, p=$ $0.013)$. The percentage of seizure spread electrodes overlying normometabolic cortex was not significantly different from that of the two other metabolic groups $(p=0.09$ and $p=0.32)$ (see Table 2).

Seizure spread occurred significantly more frequently than seizure onset over normometabolic areas (14.5 vs $4.6 \%, p=0.0078$ ), although the occurrence of seizure onset and spread was similar over both hypometabolic areas (8.9 vs $7.1 \%, p=0.80)$ and metabolic border zones (26 vs $26.2 \%, p=0.97$ ) (see Table 2 ).

All patients underwent resective surgery. Histopathological evaluation showed mild to moderate white and/or gray matter gliosis in all patients. In addition, intracellular glial inclusions were present in 2 cases, and mild cortical dysgenesis was found in 1 case. A seizure-free outcome (mean follow-up interval, $15.3 \pm$ 5.3 months; see Table 1) occurred in $8(66.7 \%)$ patients. Class III $(\mathrm{n}=2)$ and class IV $(\mathrm{n}=1)$ outcomes occurred in patients in whom the epileptic cortex as defined by intracranial EEG could not be fully resected because it involved eloquent cortex (motor areas in $\mathrm{Pa}$ tients 2 and 4 and speech area in Patient 7).

\section{Discussion}

Our study demonstrates that both seizure onset and early spread preferentially involve the border zone of hypometabolic and normometabolic cortex as well as adjacent areas with normal interictal glucose metabolism. In contrast, the most cortical areas with decreased glucose metabolism proved to be not epileptogenic as defined by intracranial EEG. This finding is at odds with the generally accepted (but unproven) concept

Table 2. Occurrence of Ictal Onset and Early Spread (as defined by invasive electroencephalography) among Electrodes Overlying Hypometabolic, Normometabolic, and Border Zone Areas

\begin{tabular}{|c|c|c|c|c|c|c|c|c|c|c|}
\hline \multirow[b]{3}{*}{ Patient No. } & \multirow{3}{*}{$\begin{array}{l}\text { Total } \\
\text { Electrodes }\end{array}$} & \multicolumn{3}{|c|}{ Hypometabolic } & \multicolumn{3}{|c|}{ Border Zone } & \multicolumn{3}{|c|}{ Normometabolic } \\
\hline & & \multirow[b]{2}{*}{ Total } & \multicolumn{2}{|c|}{ Ictal } & \multirow[b]{2}{*}{ Total } & \multicolumn{2}{|c|}{ Ictal } & \multirow[b]{2}{*}{ Total } & \multicolumn{2}{|c|}{ Ictal } \\
\hline & & & Onset & Spread & & Onset & Spread & & Onset & Spread \\
\hline 1 & 68 & 8 & $0(0 \%)$ & $0(0 \%)$ & 8 & $1(12.5 \%)$ & $0(0 \%)$ & 52 & $1(1.9 \%)$ & $10(19.2 \%)$ \\
\hline 2 & 52 & 23 & $2(8.7 \%)$ & $13(56.5 \%)$ & 21 & $4(19 \%)$ & $9(42.9 \%)$ & 8 & $0(0 \%)$ & $1(12.5 \%)$ \\
\hline 3 & 60 & 4 & $0(0 \%)$ & $0(0 \%)$ & 7 & $0(0 \%)$ & $3(42.8 \%)$ & 49 & $1(2 \%)$ & $17(34.7 \%)$ \\
\hline 4 & 64 & 17 & $0(0 \%)$ & $0(0 \%)$ & 20 & $3(15 \%)$ & $9(45 \%)$ & 27 & $0(0 \%)$ & $3(11.1 \%)$ \\
\hline 5 & 64 & 30 & $2(6.7 \%)$ & $0(0 \%)$ & 20 & $6(30 \%)$ & $4(20 \%)$ & 14 & $2(14.3 \%)$ & $0(0 \%)$ \\
\hline 6 & 68 & 0 & $0(-)$ & $0(-)$ & 12 & $2(16.7 \%)$ & $7(58.3 \%)$ & 56 & $2(3.6 \%)$ & $14(25 \%)$ \\
\hline 7 & 64 & 4 & $1(25 \%)$ & $0(0 \%)$ & 28 & $12(42.8 \%)$ & $6(21.4 \%)$ & 32 & $2(6.2 \%)$ & $2(6.2 \%)$ \\
\hline 8 & 64 & 2 & $0(0 \%)$ & $0(0 \%)$ & 11 & $2(18.2 \%)$ & $5(45.4 \%)$ & 51 & $1(2 \%)$ & $8(15.7 \%)$ \\
\hline 9 & 64 & 20 & $3(15 \%)$ & $3(15 \%)$ & 25 & $3(12 \%)$ & $7(28 \%)$ & 19 & $0(0 \%)$ & $3(15.8 \%)$ \\
\hline 10 & 64 & 0 & $0(-)$ & $0(-)$ & 4 & $2(50 \%)$ & $0(0 \%)$ & 60 & $4(6.7 \%)$ & $4(6.7 \%)$ \\
\hline 11 & 84 & 21 & $7(33.3 \%)$ & $0(0 \%)$ & 29 & $15(51.7 \%)$ & $0(0 \%)$ & 34 & $4(11.8 \%)$ & $0(0 \%)$ \\
\hline 12 & 72 & 4 & $0(0 \%)$ & $0(0 \%)$ & 9 & $4(44.4 \%)$ & $1(11.1 \%)$ & 59 & $4(6.8 \%)$ & $16(27.1 \%)$ \\
\hline $\begin{array}{l}\text { Mean ictal/ } \\
\text { total (\%) }\end{array}$ & & & 8.9 & 7.1 & & $26.0^{\mathrm{a}}$ & $26.2^{\mathrm{b}}$ & & 4.6 & 14.5 \\
\hline $\pm S D$ & & & $\pm 11.9 \%$ & $\pm 17.9 \%$ & & $\pm 17.2 \%$ & $\pm 20.5 \%$ & & $\pm 4.7 \%$ & $\pm 10.7 \%$ \\
\hline
\end{tabular}

${ }^{a}$ Mean percentage is significantly higher in this group (border zone) than among onset electrodes overlying normo- or hypometabolic cortex. ${ }^{b}$ Mean percentage is significantly higher in this group (border zone) than among electrodes with early spread overlying hypometabolic cortex. 


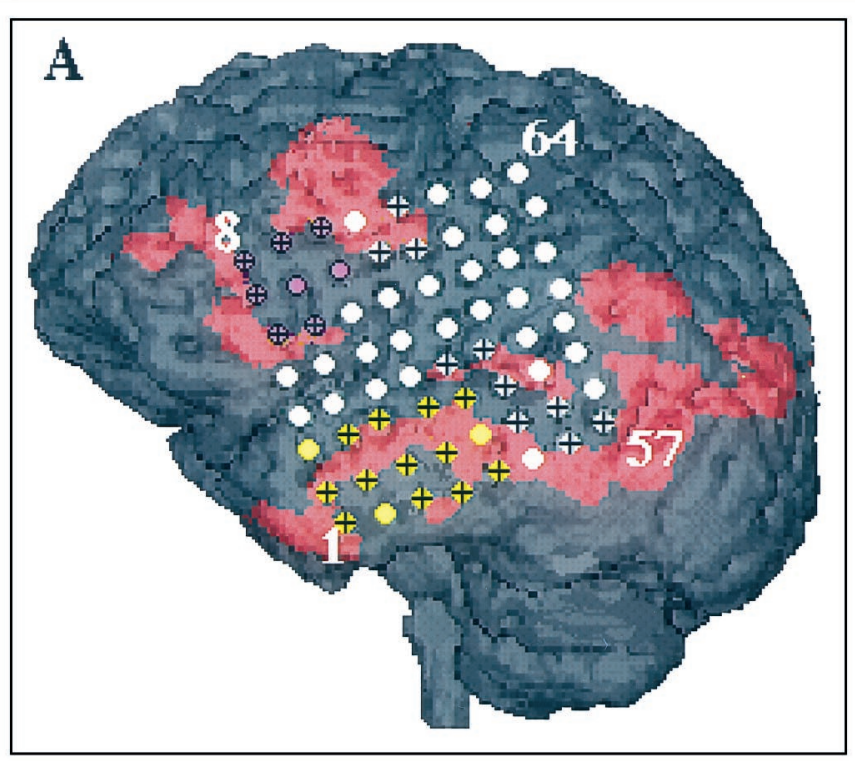

B

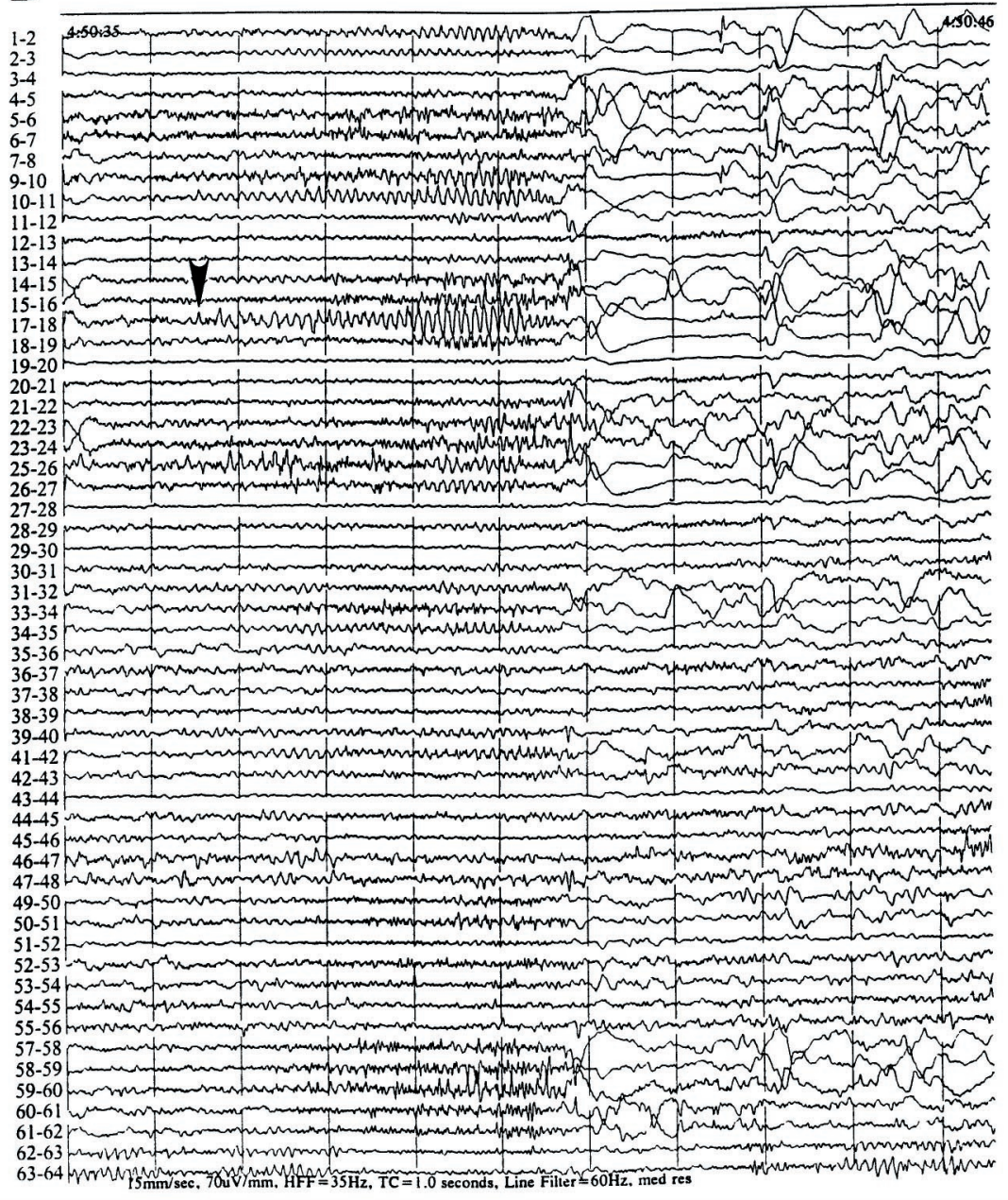

92 Annals of Neurology Vol 48 No 1 July 2000 
Fig 2. (A) Surface location of electrodes with seizure onset (yellow), early seizure spread (purple), and no early ictal involvement (white) compared with the location of underlying areas of hypometabolism (red) in Patient 7, who had a left temporal seizure onset propagating to the frontal cortex. Electrodes with a black cross were designated as overlying a metabolic border zone. The numbers represent electrode numbering of the corners of the grid. (B) Electrocorticographic data from one seizure from Patient 7 showing ictal onset. This electrocorticographic sample demonstrates the buildup of a seizure at the inferior-anterior portion of the grid (with maximum amplitude at electrodes 17 and 18; arrowhead), which mostly represents electrodes over metabolic border zones. After the sudden discontinuation of the original ictal activity, high-amplitude semirhythmic activity developed at the superior-anterior corner of the grid, which later evolved to rhythmic spiking (not shown) eventually involving a larger field. The epileptogenic temporal cortex was removed, but the frontal cortex receiving early spread was not entirely resected because of the proximity of the motor speech area. After temporary (2 months) seizure freedom, seizures originating from the frontal region recurred.

that focal areas of hypometabolism detected by interictal FDG-PET represent epileptic cortex. Our study demonstrates that ictal electrophysiological changes often tend to skip or "flow" around truly hypometabolic areas. This feature of hypometabolic cortex is remarkably similar to that of epileptogenic brain lesions (eg, tumors), where epileptic activity commonly occurs adjacent to the lesion in the surrounding normalappearing cortex. ${ }^{18,19}$

\section{Methodological Issues}

The occurrence of an electrode over hypometabolic versus normometabolic cortex or over their border zones depends on the location and size of the area designated as hypometabolic. The size of the region marked as hypometabolic is determined by the cutoff threshold applied., ${ }^{96}$ By applying a relatively low (10\%) cutoff threshold, even mild metabolic abnormalities could be detected, and the chance of designating metabolically abnormal cortex as normometabolic was minimized. Using a more rigorous criterion (higher cutoff threshold) for defining hypometabolic regions would result in smaller areas being designated as metabolically abnormal., ${ }^{9,16}$ This would further lower the rate of ictally involved electrodes overlying hypometabolic cortex, although the number of adjacent ictal electrodes would be increased (as a result of the reclassification of some border zone electrodes in the normometabolic group).

Possible errors of localization of metabolic abnormalities versus electrophysiological events could arise from inaccuracies of PET/MRI coregistration as well as from MRI/grid electrode coregistration. The maximal misregistration error for the procedures that we employed is $0.5 \mathrm{~mm}$ for PET/MRI coregistration ${ }^{15}$ and $2.7 \mathrm{~mm}$ for MRI/grid coregistration, ${ }^{12}$ which yields a possible maximum misregistration error of $3.2 \mathrm{~mm}$. Because this distance falls below the in-plane resolution of the PET images, and because it is only one third of the distance between two adjacent electrodes, electrode misregistration can be considered as a minimal source of metabolic misclassification of electrodes in this study. This is further supported by the fact that in 3 patients in whom electrode locations were determined directly from MRI with the grid in place (thus misreg- istration of MRI and the radiograph was not a source of error), a similar occurrence of electrodes with seizure involvement was found (ie, low incidence over hypometabolic and normometabolic areas and considerably higher occurrence over border zones).

Another important issue to be addressed is the interpretation of the ictal subdural EEG data. The complexity of intracranial EEG and the pitfalls of its interpretation are well known. ${ }^{20,21}$ The region of seizure onset continues to be the most reliable definition of the epileptogenic area. Although we defined early ictal spread to be within the first 10 seconds after onset, the duration after ictal onset, which should be considered significant, remains ambiguous. ${ }^{21}$ Our policy was to resect cortex giving rise to seizure onset regardless of hypometabolism on PET, cortex involved in early spread, or cortex with frequent interictal spiking (data not shown), although we spared eloquent cortex. Ideally, an electrographic pattern should be considered a reliable marker of the epileptogenic region only when a complete resection of the involved cortex correlates with seizure freedom and incomplete resection leads to persistent seizures. ${ }^{21}$ The complete resection of the epileptogenic region as defined by EEG always resulted in a good outcome (seizure-free in 8 patients and class II in 1 patient) in our group, although a poor outcome could be attributed to incomplete resection of the epileptic cortex as defined by electrocorticography as a result of the involvement of eloquent areas. These data strongly support the contention that epileptogenic areas as defined by intracranial EEG in our patients were appropriate for comparison with PET abnormalities.

\section{Possible Underlying Mechanisms of the Findings}

Possible explanations of our findings emerge from animal studies on experimental epilepsy. It has been demonstrated that cortical epileptic foci and surrounding cortical zones display different, and occasionally even opposite, electrophysiological, metabolic, and neurochemical properties. ${ }^{22-28}$ Actively spiking epileptic foci show increased glucose metabolism ${ }^{29}$ and were shown to be surrounded by widespread hypometabolic cortex extending in a dynamic fashion during the transition from interictal to ictal activity. ${ }^{26}$ It was also demonstrated that electrophysiologically hyperexcitable corti- 


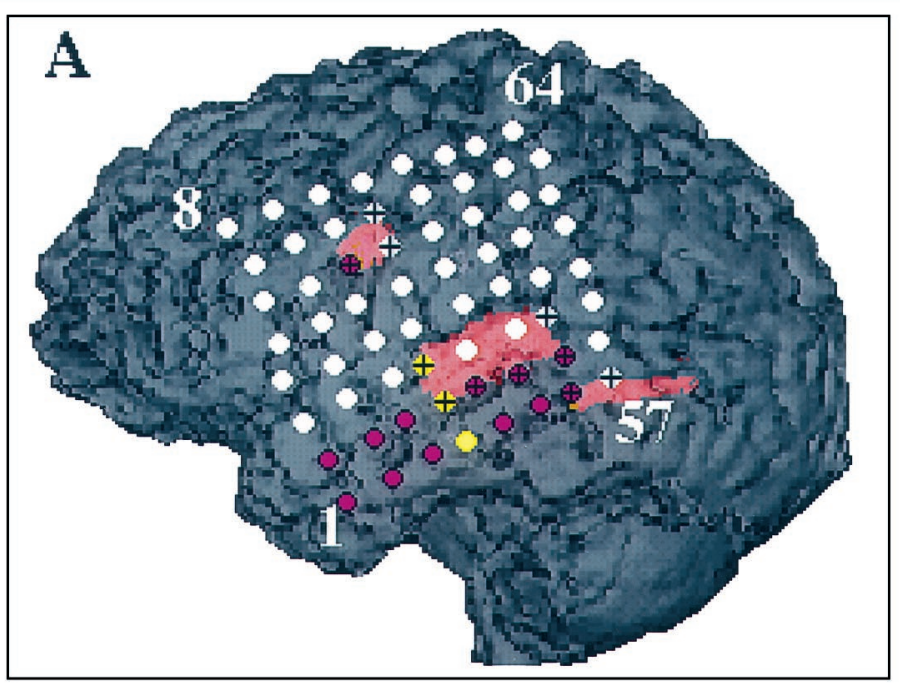

B

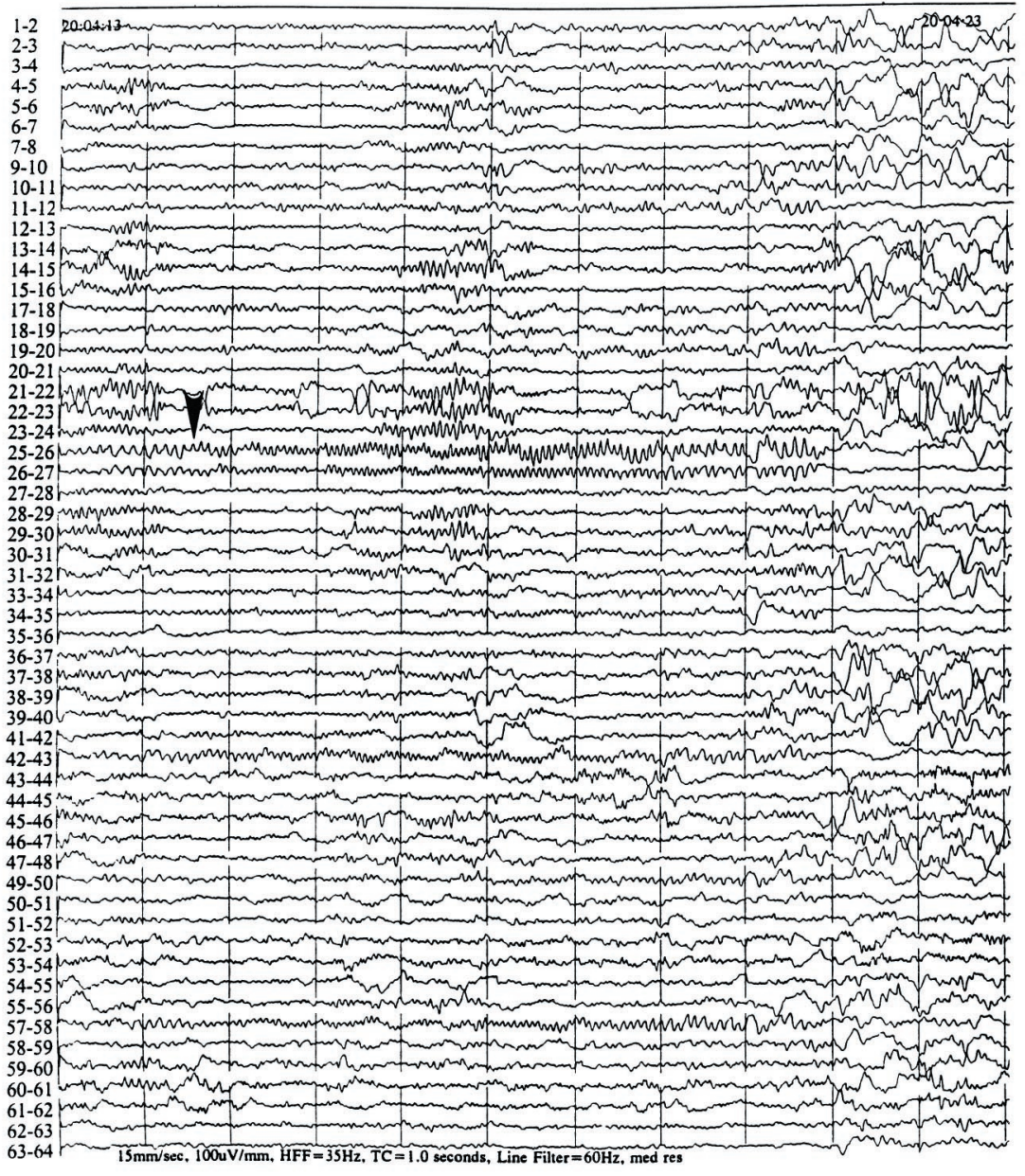

cal regions may show normal glucose metabolism in experimentally induced cortical dysplasias. ${ }^{30}$ Acutely induced focal seizure activity, however, is usually associated with increased metabolism in the focus and de- creased metabolism in ipsilateral connected areas. ${ }^{31}$ In this model, $\left[{ }^{14} \mathrm{C}\right] 2$-deoxyglucose uptake was related to the overall strength of synaptic activity, and reduced metabolism was found to be associated with decreased 


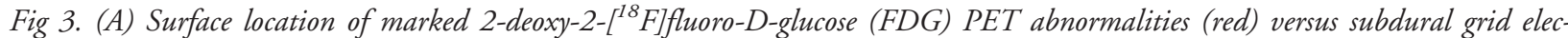
trodes (seizure onset [yellow], early seizure spread [purple], electrodes not showing early ictal involvement [white]) in Patient 8 with a left temporal onset propagating to adjacent temporal electrodes as well as frontally. (B) Electrocorticographic data from one seizure from Patient 8. Onset occurred at border zone electrodes (electrodes 25-27), whereas electrodes overlying the area of temporal hypometabolism (electrodes 35 and 43 within the red marked area on the surface picture) were not involved in seizure onset or early spread.

synaptic activity and tonic hyperpolarization of the neurons. Such electrophysiological characteristics may protect these cortical areas from ictal involvement and can represent a functional disconnection of the focus from surrounding synaptically connected areas. The functional isolation of epileptic foci from their surrounding neuronal connections may influence the excitability of this neuronal population and may prevent self-sustaining synchronized neuronal activity as well, ${ }^{32}$ as shown by successful suppression of focal epileptic activity by subpial transections both in animal models $s^{33}$ and in human epilepsy surgery of eloquent cortex. ${ }^{34,35}$ Based on these observations, one can hypothesize that hypometabolic cortex without ictal electrophysiological involvement might represent regions with functional abnormality that are actually protected from participation in the seizure activity. This hypothesis is also supported by recent findings showing a downregulation of brain-derived neurotrophic factor and other molecules in areas surrounding the epileptic focus, which likely represents an inhibitory environment that hampers seizure spread. ${ }^{27,28}$ This is consistent with our findings that although both onset and spread were equally rare over hypometabolic areas, the involvement of normometabolic areas was higher during seizure spread, suggesting that early spread occurs preferentially in normo- rather than hypometabolic regions. We suggest that the seizure focus may be inducing the PET metabolic abnormality because reversal of glucose hypometabolism in nonepileptic regions has been observed after removal of the seizure onset area in postsurgical follow-up PET studies. ${ }^{36}$

\section{Clinical Applications of the Findings}

Our findings may elucidate several controversies with regard to the use of FDG-PET in clinical epilepsy and thus may have important clinical applications. First, our findings may explain why areas of hypometabolism can occasionally miss epileptic cortex even though hypometabolism can be present in adjacent cortex that seems to be normal structurally and electrophysiologically. Our findings can also provide a feasible explanation for the commonly described discrepancy between extent of epileptic cortical regions and corresponding areas of abnormal glucose metabolism. ${ }^{6,7}$ Furthermore, these results clearly indicate that the margins of hypometabolic areas and the adjacent normometabolic cortex should be seriously considered as epileptogenic and should be addressed when subdural electrode placement is applied to capture seizures. Because the coverage of the brain surface with invasive electrodes is always limited spatially, this consideration may help to avoid sampling error, which is one of the major pitfalls of invasive EEG monitoring. ${ }^{20}$

At first glance, the findings of our study might seem to challenge the clinical usefulness of FDG-PET to detect epileptic areas; in fact, they still represent a strong spatial relation between epileptic cortex and hypometabolic regions. These findings may contribute to a better understanding of the functional link between ictal cortical electrophysiological events and the interictal abnormalities of brain glucose metabolism observed on PET scans. This may help to more reliably identify and eradicate epileptogenic brain regions in one of the most challenging groups of intractable epilepsies.

This work was supported in part by funding from NIH grant NS34488 .

We express our gratitude to Galina Rabkin, CNMT, Teresa Jones, CNMT, and Mei-li Lee, MS, for their expert technical assistance in performing the PET studies.

\section{References}

1. Debets RM, van Veelen CW, Maquet P, et al. Quantitative analysis of 18/FDG-PET in the presurgical evaluation of patients suffering from refractory partial epilepsy: comparison with CT, MRI, and combined subdural and depth EEG. Acta Neurochir Suppl (Wien) 1990;50:88-94

2. Engel J Jr, Henry TR, Risinger MW, et al. Presurgical evaluation for partial epilepsy: relative contributions of chronic depthelectrode recordings versus FDG-PET and scalp-sphenoidal ictal EEG. Neurology 1990;40:1670-1677

3. Henry TR, Sutherling WW, Engel J Jr, et al. Interictal cerebral metabolism in partial epilepsies of neocortical origin. Epilepsy Res 1991;10:174-182

4. Sadzot B, Debets RM, Maquet P, et al. Regional brain glucose metabolism in patients with complex partial seizures investigated by intracranial EEG. Epilepsy Res 1992;12:121-129

5. Theodore WH, Sato S, Kufta CV, et al. FDG-positron emission tomography and invasive EEG: seizure focus detection and surgical outcome. Epilepsia 1997;38:81-86

6. Gaillard WD, Bhatia S, Bookheimer SY, et al. FDG-PET and volumetric MRI in the evaluation of patients with partial epilepsy. Neurology 1995;45:123-126

7. da Silva EA, Chugani DC, Muzik O, Chugani HT. Identification of frontal lobe epileptic foci in children using positron emission tomography. Epilepsia 1997;38:1198-1208

8. Swartz BE, Halgren E, Delgado-Escueta AV, et al. Neuroimag- 
ing in patients with seizures of probable frontal lobe origin Epilepsia 1989;30:547-558

9. Muzik $\mathrm{O}$, da Silva EA, Juhász C, et al. Intracranial EEG vs flumazenil and glucose PET in children with extratemporal lobe epilepsy. Neurology 2000;54:171-179

10. Watson C, Jack CR Jr, Cendes F. Volumetric magnetic resonance imaging: clinical applications and contributions to the understanding of temporal lobe epilepsy. Arch Neurol 1997;54: 1521-1531

11. Engel J Jr, Van Ness PC, Rasmussen TB, Ojemann LM. Outcome with respect to epileptic seizures. In: Engel J Jr, ed. Surgical treatment of the epilepsies. New York: Raven Press, 1993; $609-621$

12. von Stockhausen HM, Thiel A, Herholz K, Pietrzyk U. A convenient method for topographical localization of intracranial electrodes with MRI and a conventional radiograph. Neuroimage 1997;5(Suppl):S514 (Abstract)

13. von Stockhausen H, Pietrzyk U, Herholz K. "3D-Tool”: a software for visualization and analysis of coregistered multimodality volume datasets of individual subjects. Neuroimage 1998; 7(Suppl):S799 (Abstract)

14. Juhász C, Nagy F, Watson C, et al. $\left[{ }^{11} \mathrm{C}\right]$ flumazenil PET in patients with epilepsy with dual pathology. Epilepsia 1999;40: 566-574

15. Pietrzyk U, Herholz K, Fink G, et al. An interactive technique for three-dimensional image registration: validation for PET, SPECT, MRI and CT brain studies. J Nucl Med 1994;35: 2011-2018

16. Muzik O, Chugani DC, Shen C, et al. Objective method for localization of cortical asymmetries using positron emission tomography to aid surgical resection of epileptic foci. Comput Aided Surg 1998;3:74-82

17. Stokking R, Zuiderveld H, Hulshoff-Pol H, Viergever M. Integrated visualization of SPECT and MR images for frontal lobe damaged regions. In: Robb E, ed. Visualization in biomedical computing. Bellingham, WA: SPIE Press, 1994:282-290

18. Engel J Jr. Intracerebral recordings: organization of the human epileptogenic region. J Clin Neurophysiol 1993;10:90-98

19. Chevassus-au-Louis N, Baraban SC, Gaïarsa JL, Ben-Ari Y. Cortical malformations and epilepsy: new insights from animal models. Epilepsia 1999;40:811-821

20. Jayakar P, Duchowny M, Resnick TJ, Alvarez LA. Localization of seizure foci: pitfalls and caveats. J Clin Neurophysiol 1991; $8: 414-431$

21. Jayakar P. Invasive EEG monitoring in children: when, where, and what? J Clin Neurophysiol 1999;16:408-418
22. Prince DA, Wilder BJ. Control mechanisms in cortical epileptogenic foci: "surround" inhibition. Arch Neurol 1967;16:194202

23. Collins RC. Use of cortical circuits during focal penicillin seizures: an autoradiographic study with $\left[{ }^{14} \mathrm{C}\right]$ deoxyglucose. Brain Res 1978;150:487-501

24. Sherwin A, Quesney F, Gauthier S, et al. Enzyme changes in actively spiking areas of human epileptic cerebral cortex. Neurology 1984;34:927-933

25. Pockberger H. Focal epilepsy: generation and spreading mechanisms in experimental conditions. Wien Klin Wochenschr 1990;102:201-205

26. Witte OW, Bruehl C, Schlaug G, et al. Dynamic changes of focal hypometabolism in relation to epileptic activity. J Neurol Sci 1994;124:188-197

27. Liang F, Jones EG. Zif268 and FOS-like immunoreactivity in tetanus toxin-induced epilepsy: reciprocal changes in the epileptic focus and the surround. Brain Res 1997;778:281-292

28. Liang F, Le LD, Jones EG. Reciprocal up- and down-regulation of BDNF mRNA in tetanus toxin-induced epileptic focus and inhibitory surround in cerebral cortex. Cereb Cortex 1998;8: 481-491

29. Bittar RG, Andermann F, Olivier A, et al. Interictal spikes increase cerebral glucose metabolism and blood flow: a PET study. Epilepsia 1999;40:170-178

30. Redecker C, Lutzenburg M, Gressens P, et al. Excitability changes and glucose metabolism in experimentally induced focal cortical dysplasias. Cereb Cortex 1998;8:623-634

31. Bruehl C, Witte OW. Cellular activity underlying altered brain metabolism during focal epileptic activity. Ann Neurol 1995; 38:414-420

32. Holmes $\mathrm{O}$. The intracortical neuronal connectivity subserving focal epileptiform activity in rat cortex. Exp Physiol 1994;79: 705-721

33. Hashizume K, Tanaka T. Multiple subpial transection in kainic acid-induced focal cortical seizure. Epilepsy Res 1998;32:389399

34. Morrell F, Whisler WW, Bleck TP. Multiple subpial transection: a new approach to the surgical treatment of focal epilepsy. J Neurosurg 1989;70:231-239

35. Smith MC. Multiple subpial transection in patients with extratemporal epilepsy. Epilepsia 1998;39(Suppl 4):S81-89

36. Hajek M, Wieser HG, Kahn N, et al. Preoperative and postoperative glucose consumption in mesiobasal and lateral temporal lobe epilepsy. Neurology 1994;44:2125-2132 